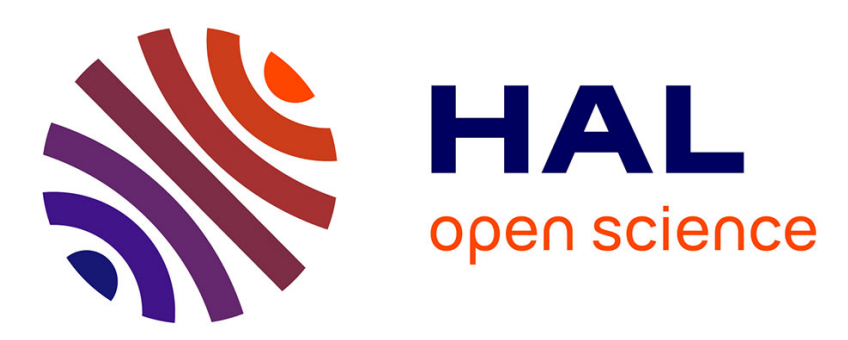

\title{
Erratum : Forme de raie pour la spectroscopie d'absorption saturée tenant compte des collisions élastiques faibles
}

Ch. J. Bordé, S. Avrillier, M. Gorlicki

\section{- To cite this version:}

Ch. J. Bordé, S. Avrillier, M. Gorlicki. Erratum: Forme de raie pour la spectroscopie d'absorption saturée tenant compte des collisions élastiques faibles. Journal de Physique Lettres, 1979, 40 (2), pp.35-35. 10.1051/jphyslet:0197900400203500 . jpa-00231563

\section{HAL Id: jpa-00231563 https://hal.science/jpa-00231563}

Submitted on 1 Jan 1979

HAL is a multi-disciplinary open access archive for the deposit and dissemination of scientific research documents, whether they are published or not. The documents may come from teaching and research institutions in France or abroad, or from public or private research centers.
L'archive ouverte pluridisciplinaire HAL, est destinée au dépôt et à la diffusion de documents scientifiques de niveau recherche, publiés ou non, émanant des établissements d'enseignement et de recherche français ou étrangers, des laboratoires publics ou privés. 


\section{ERRATUM}

\section{Forme de raie pour la spectroscopie d'absorption saturée tenant compte des collisions élastiques faibles}

Ch. J. Bordé, S. Avrillier et M. Gorlicki

Laboratoire de Physique des Lasers, avenue J.-B.-Clément, 93430 Villetaneuse, France.

(J. Physique Lett. 38 (1977) L-249)

Les équations (5) et (7) doivent être remplacées par les suivantes :

$$
\begin{aligned}
{\left[\frac{\partial}{\partial t^{\prime}}+\gamma_{\alpha}-\tilde{W}_{\alpha}(\theta)\right] \tilde{n}_{\alpha}^{\prime}\left(\mathbf{r}^{\prime}, t^{\prime}, \mathbf{v}_{\perp}, \theta\right)=\left[\gamma_{\alpha}\right.} & \left.-\tilde{W}_{\alpha}(\theta)\right] \tilde{n}_{\alpha}^{\prime(0)}\left(\mathbf{v}_{\perp}, \theta\right)+ \\
& +i \varepsilon_{\alpha}\left\{\sum_{j, \pm} \Omega_{j}^{ \pm} \mathrm{e}^{-i \varphi_{j}^{ \pm}} U_{j}^{ \pm *}\left(\mathbf{r}_{\perp}^{\prime}+\mathbf{v}_{\perp} t^{\prime}, z\right)\left(\tilde{\rho}_{\mathrm{ba}}^{\prime \pm}\left(\mathbf{r}^{\prime}, t^{\prime}, \mathbf{v}_{\perp},-\theta \mp t^{\prime}\right)\right)^{*}-\right. \\
& \left.-\sum_{j, \pm} \Omega_{j}^{ \pm} \mathrm{e}^{i \varphi \frac{ \pm}{j}} U_{j}^{ \pm}\left(\mathbf{r}_{\perp}^{\prime}+\mathbf{v}_{\perp} t^{\prime}, z\right) \tilde{\rho}_{\mathrm{ba}}^{\prime \pm}\left(\mathbf{r}^{\prime}, t^{\prime}, \mathbf{v}_{\perp}, \theta \mp t^{\prime}\right)\right\}
\end{aligned}
$$

$$
\begin{aligned}
\tilde{n}_{\alpha}\left(\mathbf{r}, t, \mathbf{v}_{\perp}, \theta\right)-\widetilde{n}_{\alpha}^{(0)}\left(\mathbf{v}_{\perp}, \theta\right)=i \varepsilon_{\alpha} & \left\{\sum_{j, \pm} \Omega_{j}^{ \pm} \mathrm{e}^{-i \varphi_{j}^{ \pm}} \int_{0}^{+\infty} \mathrm{d} \tau \widetilde{G}_{\alpha}(\theta, \tau) U_{j}^{ \pm *}\left(\mathbf{r}-\mathbf{v}_{\perp} \tau\right)\left(\tilde{\rho}_{\mathrm{ba}, \pm 1}\left(\mathbf{r}-\mathbf{v}_{\perp} \tau, t-\tau, \mathbf{v}_{\perp},-\theta\right)\right)^{*}-\right. \\
& \left.-\sum_{j, \pm} \Omega_{j}^{ \pm} \mathrm{e}^{i \varphi_{j}^{ \pm}} \int_{0}^{+\infty} \mathrm{d} \tau \widetilde{G}_{\alpha}(\theta, \tau) U_{j}^{ \pm}\left(\mathbf{r}-\mathbf{v}_{\perp} \tau\right) \tilde{\rho}_{\mathrm{ba}, \pm 1}\left(\mathbf{r}-\mathbf{v}_{\perp} \tau, t-\tau, \mathbf{v}_{\perp}, \theta\right)\right\}
\end{aligned}
$$

Ces formules conduisent pour $\theta=0$ au même résultat final qui est donc inchangé. Nous signalons également une erreur typographique dans la formule (13) :

$$
\operatorname{sinc}\left(k v_{z} \tau\right) \text { est la fonction sinus cardinal de l'argument } k v_{z} \tau \text {. }
$$

